\title{
131. On Certain Identities between the Traces of Hecke Operators
}

\author{
By Hiroaki HiJIKATA
}

(Comm. by Kunihiko KodaIRA, M. J. A., Oct. 12, 1972)

Linear relations between the traces of Hecke operators and those of the Brandt matrices were first obtained by Eichler [1] and [2], then generalized by Shimizu [6]. In this note, we shall further generalize (with respect to the levels of the groups involved) and in a sense sharpen (by restricting the operators to the essential parts) their results.

Let $\boldsymbol{Z}, \boldsymbol{Q}, \boldsymbol{R}$ and $\boldsymbol{C}$ denote the set of integers, rational numbers, real numbers and complex numbers respectively. For a prime $p$, let $\boldsymbol{Z}_{p}$ and $\boldsymbol{Q}_{p}$ denote the set of $p$-adic integers and $p$-adic numbers. For a ring $M$ with a unity, let $M^{\times}$denote the group of invertible elements of $M$.

Let $B$ be a quaternion algebra having $Q$ as its center. Let $d^{2}$ be its discriminant, i.e. $d$ is a product of all distinct primes $p$ where the completion $B_{p}=B \otimes Q_{p}$ is a division algebra. We admit the case where $d=1$, namely $B$ is the two by two total matrix algebra $M_{2}(Q)$. Let $N$ be a product of $d$ and a natural number $M$ prime to $d, N=d M,(d, M)=1$. An order $I$ of $B$ is called a split order of level $N$, if it satisfies (i) if $p \mid d, \boldsymbol{I}_{p}=\boldsymbol{I} \otimes Z_{p}$ is a maximal order of $B_{p}$, and (ii) if $(p, d)=1$, there is an isomorphism $\varphi_{p}: B_{p} \rightarrow M_{2}\left(\boldsymbol{Q}_{p}\right)$ over $\boldsymbol{Q}_{p}$ such that $\varphi_{p}\left(I_{p}\right)=\left(\begin{array}{cc}\boldsymbol{Z}_{p} & \boldsymbol{Z}_{p} \\ M \boldsymbol{Z}_{p} & \boldsymbol{Z}_{p}\end{array}\right)$. Now we fix a split order $I$ of level $N$, and an isomorphism $\varphi_{p}: B_{p} \rightarrow M_{2}\left(\boldsymbol{Q}_{p}\right)$ for each $p$ prime to $d$, and write $\varphi_{p}(x)=\left(\begin{array}{ll}a_{p}(x) & b_{p}(x) \\ c_{p}(x) & d_{p}(x)\end{array}\right)$ for $x \in B_{p}$. In the following assume that $B$ is indefinite unless otherwise stated, and fix an isomorphism $\varphi: B \otimes R \rightarrow M_{2}(R)$. Let $I^{1}$ denote the group of all elements of reduced norm 1 in $\boldsymbol{I}$. Let $\Gamma=\Gamma(\boldsymbol{I})=\varphi\left(\boldsymbol{I}^{1}\right)$, and we identify $\Gamma$ with $\boldsymbol{I}^{1}$, when convenient. Then $\Gamma$ is a subgroup of the connected component $G L_{2}^{+}(\boldsymbol{R})$ of $G L_{2}(\boldsymbol{R})$. The group $G L_{2}^{+}(\boldsymbol{R})$ is acting on the complex upper half plane $\boldsymbol{H}$ as linear fractional transformations. Under this action, $\boldsymbol{H} / \Gamma$ has a finite invariant volume, and it is compact if and only if $d>1$.

Let $c$ be a divisor of $M$, and $\chi:(Z / c Z)^{\times} \rightarrow C^{\times}$be a primitive character modulo $c$. Let $\Delta$ be the subset of $I$ consisting of all elements $x$ such that $a_{p}(x) \not \equiv 0 \bmod p$ for any prime $p$ dividing $M$. Starting from the given character $\chi$, let us define the map $\chi: \Delta \rightarrow C^{\times}$by the formula $\chi(x)=\prod_{p \mid c} \chi\left(a_{p}(x)\right)$ for $x \in \Delta$. This new $\chi$ is multiplicative on $\Delta$, and its 
restriction to $\Gamma$ is a linear character of $\Gamma$. Let $\Gamma_{\chi}$ be the kernel of $\chi$ in $\Gamma$, and consider the space $S_{k}\left(\Gamma_{\chi}\right)$ of cusp forms of weight $k$ with respect to $\Gamma_{\chi}$ in the sense of [7] p. 30. Let $S_{k}(\Gamma, \chi)$ denote the set of all elements of $S_{k}\left(\Gamma_{\chi}\right)$ satisfying

$$
f \mid[\gamma]_{k}=\chi(\gamma)^{-1} f \quad \text { for all } \gamma \in \Gamma \text {, }
$$

where in general, $f \mid[g]_{k}=\operatorname{det} g^{k / 2} f(g(z))(c z+d)^{-k}$ for any

$$
g=\left(\begin{array}{ll}
a & b \\
c & d
\end{array}\right) \in G L_{2}^{+}(R) .
$$

Let $\boldsymbol{I}^{\prime}$ be a split order containing $\boldsymbol{I}$ and its level $N^{\prime}$ is divisible by $c$. Using the same $\varphi_{p}$ and $\varphi$, we define $\chi: \Delta\left(\boldsymbol{I}^{\prime}\right) \rightarrow C^{\times}$and $\Gamma\left(\boldsymbol{I}^{\prime}\right)=\varphi\left(\left(\boldsymbol{I}^{\prime}\right)^{1}\right)$. Then $S_{k}\left(\Gamma\left(I^{\prime}\right), \chi\right)$ can be considered as a subspace of $S_{k}(\Gamma, \chi)$. Let $S^{\prime}$ denote the subspace of $S_{k}(\Gamma, \chi)$ spanned by all $S_{k}\left(\Gamma\left(\boldsymbol{I}^{\prime}\right), \chi\right)$ such that $\boldsymbol{I}^{\prime}$ is a split order properly containing $I$ and its level is divisible by $c$. Let $S_{k}^{0}(\Gamma, \chi)$ denote the orthogonal complement of $S^{\prime}$ in $S_{k}(\Gamma, \chi)$ with respect to the Petersson metric, and call it the essential part of $S_{k}(\Gamma, \chi)$.

Consider the Hecke ring $R(\Gamma, \Delta)$ in the sense of [7] p. 54. Let $T_{n}=\sum \Gamma \alpha \Gamma$ denote the element of $R(\Gamma, \Delta)$ defined as the sum of all the distinct double-cosets $\Gamma \alpha \Gamma$ such that $\alpha \in \Delta$ and the reduced norm $\operatorname{Nr}(\alpha)=n$. Define the representation $\rho: R(\Gamma, \Delta) \rightarrow$ End $S_{k}(\Gamma, \chi)$ as in [7], i.e.

$$
\rho(\Gamma \alpha \Gamma): f \mapsto(\operatorname{det} \alpha)^{k / 2-1} \sum_{\nu} \chi\left(\alpha_{\nu}\right) f \mid\left[\alpha_{\nu}\right]_{k}
$$

for $\Gamma \alpha \Gamma=\bigcup_{\nu} \Gamma \alpha_{\nu}$ (disjoint), and

$$
\rho(T)=\sum c_{\alpha} \rho(\Gamma \alpha \Gamma) \quad \text { for } T=\sum c_{\alpha} \Gamma \alpha \Gamma .
$$

The essential part $S_{k}^{0}(\Gamma, \chi)$ is invariant under $\rho(T)$ for any $T \in R(\Gamma, \Delta)$. Let $\rho_{0}$ denote the restriction of $\rho$ on $S_{k}^{0}(\Gamma, \chi)$.

If $d=1$, by choosing $\varphi_{p}$ and $\varphi$ to be the natural injections, we can identify $\Gamma$ as $\Gamma_{0}(N)=\left\{\left(\begin{array}{ll}a & b \\ c & d\end{array}\right) \in S L_{2}(Z) ; c \equiv 0 \bmod N\right\}$. Then the operator $\rho\left(T_{n}\right)$ is of course, the operator $T_{n}^{N}$ of Hecke.

If $B$ is definite, we shall redefine everything. However, our previous work [4] treated (essentially) the same problem for definite quaternions, and the corresponding notions are defined there under slightly more restricted setting. So we omit the detail, and simply give the correspondence. Namely, take $S_{k}(\Gamma, \chi)=S_{k}(I)$ of [4] where only the case $\chi=1$ is discussed, and $S_{k}^{0}(\Gamma, \chi)=\boldsymbol{S}_{k}^{0}(I), T_{n}=\boldsymbol{T}(n), \rho=\rho$ of [4], respectively, and put $\rho_{0}=$ the restriction of $\rho$ on $\boldsymbol{S}_{k}^{0}(\boldsymbol{I})$.

Let $H(I)$ denote the subring of $R\left(I^{1}, \Delta\right)$ generated by all $T_{n}$ 's. It is basic and is easily seen that the structure of $H(I)$ depends only on the level $N$ of $\boldsymbol{I}$. Namely if $\boldsymbol{I}^{\prime}$ is another split order of level $N$ in some quaternion $B^{\prime}$, and $T_{n}\left(I^{\prime}\right)$ be the element of $R\left(I^{\prime 1}, \Delta^{\prime}\right)$ corresponding to $T_{n}$, then there is an isomorphism $H(I) \rightarrow H\left(I^{\prime}\right)$ such that $T_{n} \mapsto T_{n}\left(I^{\prime}\right)$. Thus we can consider $\rho_{0}=\rho_{0}(I, \chi)$ obtained from various $I$ and $\chi$, as a 
representation of one and the same ring $H(N)$ uniquely determined by $N$. $H(N)$ is a commutative ring and the image $\rho_{0}(H(N))$ is a semisimple ring. Hence $\rho_{0}=\rho_{0}(\boldsymbol{I}, \chi)$ is equivalent to $\rho_{0}^{\prime}=\rho\left(\boldsymbol{I}^{\prime}, \chi^{\prime}\right)$ if and only if the trace coincides, i.e. $\operatorname{tr} \rho_{0}\left(T_{n}\right)=\operatorname{tr} \rho_{0}^{\prime}\left(T_{n}\right)$ for any $n$. Apparently $\operatorname{tr} \rho_{0}\left(T_{n}\right)$ depends on $I, \chi$ and, to be precise, on the choice of $\varphi_{p}$ and $\varphi$.

Theorem 1. If $(n, N)=1$, then $\operatorname{tr} \rho_{0}\left(T_{n}\right)$ depends only on $n, N$ and $\chi$. In particular, whatever a quaternion $B$ and a split order $I$ of $B$ should we take, the trace $\operatorname{tr} \rho_{0}\left(T_{n}\right)$ is equal to the trace of $T_{n}^{N}$ on $S_{k}^{0}\left(\Gamma_{0}(N), \chi\right)$ as far as the level of $\boldsymbol{I}$ is $N$.

In the way of proof of the theorem, we shall show

Corollary 1. $S_{k}(\Gamma, \chi)$ is the direct sum of the spaces $S_{k}^{0}\left(\Gamma\left(\boldsymbol{I}^{\prime}\right), \chi\right)$ of all split order $\boldsymbol{I}^{\prime}$ containing $\boldsymbol{I}$ and the level is divisible by the conductor $c$ of $\chi$.

Outline of proof. Let $\boldsymbol{I}^{\prime}$ be a split order of level $N^{\prime}$ such that $\boldsymbol{I} \subset \boldsymbol{I}^{\prime}$ and $c \mid N^{\prime}$. Put $N=c d A$ and $N^{\prime}=c d a, a \mid A$. Since $(n, N)=1$, $\rho\left(T_{n}\right)$ keeps $S_{k}\left(\Gamma\left(\boldsymbol{I}^{\prime}\right), \chi\right)$ and $S_{k}^{0}\left(\Gamma\left(\boldsymbol{I}^{\prime}\right), \chi\right)$ keeps invariant. Let $\rho^{\prime}\left(T_{n}\right)$ (resp. $\rho_{0}^{\prime}\left(T_{n}\right)$ ) denote the restriction of $\rho\left(T_{n}\right)$ on $S_{k}\left(\Gamma\left(I^{\prime}\right), \chi\right)$ (resp. $\left.S_{k}^{0}\left(I^{\prime}\right), \chi\right)$ ). If we fix $\chi$, then it is rather obvious that $\operatorname{tr} \rho^{\prime}\left(T_{n}\right)$ (resp. tr $\left.\rho_{0}^{\prime}\left(T_{n}\right)\right)$ depends only on $d, n$ and $a$, so fixing $d$, we can put $\lambda_{a}(n)$ $=\operatorname{tr} \rho^{\prime}\left(T_{n}\right), \lambda_{a}^{0}(n)=\operatorname{tr} \rho_{0}^{\prime}\left(T_{n}\right) . \quad$ In particular, $\quad \lambda_{A}(n)=\operatorname{tr} \rho\left(T_{n}\right), \lambda_{A}^{0}(n)$ $=\operatorname{tr} \rho_{0}\left(T_{n}\right)$. If Corollary 1 is true, since the number of $\boldsymbol{I}^{\prime}$ having level $N^{\prime}$ is equal to the number of divisors $D(A / a)$ of $A / a$, we should have

$$
\lambda_{A}(n)=\sum_{a \mid A} D(A / a) \lambda_{a}^{0}(n) .
$$

Hence $\lambda_{A}^{0}(n)=\sum_{a \mid A} \beta(A / a) \lambda_{a}(n)$ with $\beta(m)=\sum_{a \mid m} \mu(m / a) \mu(a)$ by the Möbius function $\mu(\quad)$. Now, by the formula of the traces given in [3], we can prove

Lemma. $\quad \sum_{a \mid A} \beta(A / a) \lambda_{a}(n)$ depends only on $n, N$ and $\chi$ (independent of $d$ !).

Then Theorem 1 has been proved if $A$ is a prime. We can proceed by induction on $A$, by the similar way in the proof of Theorem 2 of [4] plus the approximation theorem, and prove Corollary 1, hence simultaneously Theorem 1.

Corollary 2. For any $n, \operatorname{tr} \rho_{0}\left(T_{n}\right)$ depends only on $n, N$ and $\chi$.

The proof depends on a careful observation based on Theorem 1 .

As an application, we can generalize a theorem of Hecke, AtkinLehner and T. Miyake [5] on $\Gamma_{0}(N)$ to the quaternion groups $\Gamma(\boldsymbol{I})$. Namely, let $L$ denote the ring generated by all $\rho\left(T_{n}\right)$ and their adjoint $\rho\left(T_{n}\right)^{*}$, then

Theorem 2. $S_{k}^{0}(\Gamma(I), \chi)$ is the largest L-invariant subspace of $S_{k}(\Gamma(I), \chi)$ on which $L$ is semi-simple and commutative.

Let $\boldsymbol{I}_{j}(j=1,2)$ be split orders containing $\boldsymbol{I}$, with the levels divisible by the conductor $c$ of $\chi$. Let $f_{j} \in S_{k}^{0}\left(\Gamma\left(\boldsymbol{I}_{j}\right), \chi\right)$ be a non-zero common 
eigen function of all $\rho\left(T_{p}\right)$ such that $(p, N)=1$. Let $a_{j}(p)$ be the eigen value of $\rho\left(T_{p}\right)$. Then $a_{1}(p) \neq a_{2}(p)$ for all but a finite number of $p$ 's, unless $f_{1}$ is a constant multiple of $f_{2}$.

A proof of the former part needs some computation. The latter part is immediate from Theorem 1 and the result of [5].

\section{References}

[1] M. Eichler: Über die Darstellbarkeit von Modulformen durch Thetareihen. J. Reine Angew. Math., 195, 156-171 (1956).

[2] —_: Quadratische Formen und Modulfunktionen. Acta Arith., 4, 217239 (1958).

[3] H. Hijikata: Explicit formula of traces of Hecke operators for $\Gamma_{0}(N)$ (to appear in J. of Math. Soc. Jap.).

[4] H. Hijikata and H. Saito: On the Representability of Modular Forms by Theta Series (to appear in Number Theory, Algebraic Geometry and Commutative Algebras in Honour of Yasuo Akizuki, Kinokuniya, Tokyo, 1973).

[5] T. Miyake: On automorphic forms on $G L_{2}$ and Hecke operators. Ann. of Math., 94, 174-189 (1971).

[6] H. Shimizu: On zeta functions of quaternion algebras. Ann. of Math., 81, 166-193 (1965).

[7] G. Shimura: Arithmetic Theory of Automorphic Functions. Publications of Math. Soc. Jap., 1971. 